\title{
Análise dos modelos colaborativos de softwares para edição de ontologias por meio do Modelo 4C de Colaboração
}

\author{
Marcel Ferrante Silva \\ Doutor; Universidade Federal de Goiás, Goiânia, GO, Brasil; \\ marcelf@gmail.com \\ Dalton Lopes Martins \\ Doutor; Universidade de Brasília, Brasília, DF, Brasil; \\ dmartins@gmail.com \\ Joyce Siqueira \\ Mestra; Universidade Federal de Goiás, Goiânia, GO, Brasil; \\ joycitta@gmail.com \\ Douglas Veronez Santana \\ Graduando; Universidade Federal de Goiás, Goiânia, GO, Brasil; \\ douglasveronezz@gmail.com
}

\begin{abstract}
Resumo: Esta pesquisa objetiva apresentar os modelos de colaboração de seis softwares editores de ontologia: MusicBrainz, OntoWiki, Semantic MediaWiki, Tainacan Ontology, WebProtégé e Wikidata, e relacionar as ferramentas para colaboração disponíveis classificando-as de acordo com o Modelo 4C de Colaboração, que abrange a comunicação, coordenação e colaboração e cooperação. O resultado abrange diversas nuances em relação aos softwares, detalhando a participação em cada ' $\mathrm{C}$ ' do Modelo, mas de forma geral, apresenta o WebProtégé com o maior número de quesitos de comunicação, enquanto o Tainacan Ontology possui o maior número de quesitos relacionados à coordenação e a colaboração e cooperação. O modelo de colaboração dos softwares é essencial para promover a interação, de forma a mediar as divergências e possibilitar efetiva colaboração, por isso, quanto mais completo, melhor é o serviço oferecido, assim, espera-se que estes resultados sirvam como base para atualizações dos softwares existentes ou para criação de novos.
\end{abstract}

Palavras-chaves: Modelo 4C de Colaboração. Modelo de Colaboração. Softwares Editores de Ontologias.

\section{Introdução}

Ontologias são uma especificação formal explícita de uma conceitualização compartilhada, isto é, uma abstração de algum conceito ou fenômeno que classifica e define tipos e limitações de uso. Sua representação deve ser interpretada e processada por uma máquina a fim de capturar e estruturar um conhecimento (GRUBER, 1995). Especificamente para organização e 
representação do conhecimento, o sentido é diferente, visto que a ontologia se propõe a classificar as coisas em categorias, na perspectiva do sujeito e da linguagem do domínio (VITAL; CAFÉ, 2011).

Dessa forma, gerir ontologias tornou-se ponto alvo para o desenvolvimento de softwares, que deveriam ser capazes de incluir, editar e gerenciar informações de forma colaborativa, o que também culmina na concepção da Web 2.0. No entanto, ressalta-se que o trabalho colaborativo implica, muitas vezes, em divergências entre os participantes, principalmente pelos diferentes pontos de vista existentes. Assim, torna-se vital que os softwares contemplem recursos para finalização de disputas de conteúdo e, até mesmo, normas para nortear a conduta dos usuários.

Considerando o contexto supramencionado, essa pesquisa visa apresentar e avaliar o modelo de colaboração de seis softwares editores de ontologias: MusicBrainz, OntoWiki, Semantic MediaWiki, Tainacan Ontology, WebProtégé e Wikidata, utilizando as premissas do Modelo 4C de Colaboração, para obter, ao final, dados que quantifiquem e qualifiquem os softwares, elencando e discutindo quais podem oferecer melhores condições para colaboração entre usuários.

O Modelo 4C é uma evolução do Modelo 3C, no qual foi adicionado o termo 'colaboração', ficando, assim, o trabalho colaborativo compreendido em 3 pilares: comunicação, coordenação e colaboração e cooperação. É um modelo útil para o desenvolvimento de sistemas colaborativos e define que: a comunicação compreende a troca de mensagens e a negociação de compromissos; a coordenação compreende a gestão de pessoas, tarefas e recursos para lidar com conflitos de interesse; e a colaboração e cooperação compreende tarefas desenvolvidas em conjunto (colaboração) ou individualmente (cooperação), mas com um objetivo comum, por meio de um espaço partilhado (COSTA; LOUREIRO; REIS, 2014).Nos últimos anos, os Modelos 3C e 4C vêm sendo utilizados para avaliação de softwares colaborativos, dos quais se cita as pesquisas de: Castro, Gonçalves e Figueiredo (2017), visaram identificar as características e funcionalidades dos sites do tipo meal sharing; Melo et al. (2017), apresentaram uma ferramenta em forma de 
repositório colaborativo que pudesse servir de guia para os desenvolvedores seguindo boas práticas relacionadas a interfaces destinadas a usuários autistas; Cruz Junior et al. (2015), objetivaram identificar e analisar as principais funcionalidades colaborativas do Círculo do Coração (CirCor); Bassani, Reis e Dalanhol (2016), visaram analisar os repositórios de atividades de aprendizagem, a fim de identificar as possibilidades de compartilhamento dessas atividades e os recursos de colaboração presentes nestes utilizando diferentes Modelos, o 3C, o 4C (conexão) e o Enterprise 2.0; Costa et al. (2015), objetivaram descrever o modelo $4 \mathrm{C}$ e a sua aplicabilidade na análise da Metodologia Híbrida de Desenvolvimento Centrado no Utilizador e Costa, Reis e Loureiro (2014), que, com base nas dimensões do modelo 4C, realizaram a análise das interações entre os elementos da equipe multidisciplinar que desenvolveu o recurso educativo Courseware Sere, relativamente à dimensão Coordenação.

Nas próximas seções são apresentadas informações relevantes para compreensão do objetivo desta pesquisa. Na seção 2, a Metodologia; na 3, Resultados e Discussões, que apresenta a descrição dos modelos colaborativos e a análise do Modelo 4C de Colaboração e por último, na 4, as Considerações finais.

\section{Metodologia}

Quanto à natureza, esta pesquisa trata-se de um trabalho original. Quanto ao objetivo, mostra-se exploratória e aplicada. Quanto à abordagem, qualitativa (SEVERINO, 2017).

Buscou-se, a partir de revisão bibliográfica e experimento nos softwares, descrever o modelo de colaboração de: MusicBrainz, OntoWiki, Semantic MediaWiki, Tainacan Ontology, WebProtégé e Wikidata, para, na sequência, classificar suas ferramentas de acordo com o Modelo 4C de Colaboração (COSTA; JOÃO LOUREIRO; REIS, 2014), uma evolução do Modelo 3C de Colaboração (FUKS et al., 2005), e mensurar e discutir os resultados obtidos. 
O modelo 4C difere do modelo 3C de colaboração pelo fato de se considerar que os conceitos de colaboração e cooperação são distintos. Na literatura é recorrente os termos colaboração e cooperação surgirem como sinónimos. Na realidade são conceitos diferentes, existindo apenas um fator que é análogo: os elementos trabalham para atingirem um objetivo comum (COSTA et al., 2015, p. 22).

Os referidos softwares foram selecionados a partir de pesquisa realizada na base de dados Google Scholar, utilizando as strings de busca: "software editor de ontologias" e "ontology software editor", ambas com o filtro por período ativado, restringindo as buscas aos últimos 11 anos (2007 - 2017). Inicialmente, listou-se quais eram os softwares mais relevantes e ativos. A partir dessa lista, elencou-se os que utilizam plataforma web, são open source e que oferecem algum tipo de colaboração, resultando assim, nos softwares avaliados nesta pesquisa.

Além da classificação, as ferramentas colaborativas utilizadas pelos softwares estão especificadas e definidas no Apêndice A.

\section{Resultados e discussões}

Nesta seção são apresentadas as descrições dos modelos colaborativos dos softwares, assim como o resultado da análise do Modelo 4C de colaboração.

\subsection{Modelos Colaborativos}

A Web 2.0 possibilita aos usuários evidenciar seus conhecimentos, interagir com outros usuários e obter novas perspectivas que, combinadas a outras informações, constroem o conhecimento coletivo, sem, contudo, possuir conhecimento avançado em computação. Como principais exemplos cita-se: blogs, redes sociais, wikis, sites de compartilhamento de conteúdo, social bookmarks, fóruns e editores colaborativos de documentos (OLIVEIRA; DUTRA, 2014).

Nesta pesquisa, o enfoque está nos editores colaborativos de ontologias, especificamente os softwares MusicBrainz, OntoWiki, Semantic MediaWiki, 
Tainacan Ontology, WebProtégé e Wikidata, que estão apresentados a seguir junto a seu modelo de colaboração.

Todas as ferramentas supracitadas possibilitam a edição de ontologias, porém, cada uma com um contexto de uso diferente. Nesse ponto é importante frisar que, embora todas possibilitem a edição de ontologias, apenas três delas tem essa função como o objetivo principal, sendo elas: OntoWiki, WebProtégé e Tainacan Ontology. As demais, MusicBrainz, Semantic MediaWiki e Wikidata, apenas oferecem essa possibilidade porque estruturam os dados semanticamente.

\subsubsection{MusicBrainz}

O software tem como objetivo coletar metadados de músicas e torná-los acessíveis aos usuários, permitindo que qualquer pessoa divulgue dados que estejam sob uma licença aberta. Uma de suas principais características é a confiabilidade dos dados, sem ambiguidade. Isso porque a inserção e controle são realizados por um conjunto de editores voluntários, que obedecem a um Código de Conduta que fornece informações sobre como se portar em situações de conflitos de conteúdo (MUSICBRAINZ, 2017c).

Existem quatro tipos de editores: os Auto-editors, que são selecionados com base no seu conhecimento aprofundado em um determinado artista ou gênero musical e cujas edições não passam pelo processo de votação, além disso, podem aprovar edições de terceiros; os Relationship Editors, que podem editar tipos de relacionamento de entidades e atributos de entidades; os Transclusion Editors, que editam a documentação do sistema e por fim, os Location Editors, que adicionam e editam áreas geográficas relacionadas às entidades da base de conhecimento (SANTANA et al., 2018).

Para os demais usuários registrados é permitido: editar todas as alterações, que, para se tornarem permanentes, passam por um processo de votação, e cadastrar releases e Disc IDs, sendo que, Disc ID é uma identificação única para um determinado $\mathrm{CD}$ e o release uma versão específica de um álbum lançado (SANTANA et al., 2018. 
As edições ficam abertas para votação por sete dias. Ao final é realizada uma checagem: se a quantidade de votos positivos for maior que os negativos, a edição é aplicada, caso contrário, rejeitada (MUSICBRAINZ, 2017b).

Em relação aos mecanismos de colaboração, um dos principais é a lista de e-mail, que tem o propósito de disponibilizar meios para comunicação a respeito de assuntos gerais, incluindo temas como: interpretar (Users mailing list), sugerir alterações ou relatar problemas nas diretrizes de estilo (Style mailing list) e discutir assuntos técnicos a respeito do desenvolvimento do MusicBrainz e de suas bibliotecas (Developers mailing list). São também utilizadas para feed de notícias, bugs e commits e há a opção de se inscrever para um determinado artista ou conjunto de artistas, e assim, receber suas atualizações por $e$-mail (SANTANA et al., 2018; MUSICBRAINZ, 2017a).

O Código de Conduta também define alguns parâmetros éticos sobre a interação dos usuários no sistema, que, de forma geral, preza que os usuários se portem de maneira educada e respeitosa.

\subsubsection{OntoWiki}

O sistema OntoWiki preza pela apresentação das informações de maneira estruturada, embora possua elementos com comportamento semelhante ao de uma wiki. Entre suas funcionalidades lista-se: os mecanismos para edição de conteúdo; registro de alterações na ontologia; reversão de alterações e colaboração multiusuário. Dessa forma, as ferramentas que promovem a colaboração são (AUER; DIETZOLD; RIECHERT, 2006):

a) commenting, para permitir que usuários comentem para entidades específicas da ontologia;

b) history, para reverter uma determinada mudança realizada;

c) activity/provenance, para gratificar usuários ativos do sistema ou em uma base de conhecimento específica. 


\subsubsection{Semantic MediaWiki}

Semantic MediaWiki (SMW) é um sistema que implementa uma wiki, funcionando como extensão para o MediaWiki, software open source implementado com tecnologias da web semântica: OWL, RDF (SEMANTIC MEDIAWIKI, 2017).

Fornece uma linguagem de marcação própria, dessa forma, o usuário pode configurar elementos semânticos, tais como: classes, propriedades e relacionamentos, apresentando, assim, o conteúdo de uma wiki em forma de texto em páginas web (SANTANA et al., 2018).

Em se tratando das ferramentas de colaboração, o SMW parte do princípio da boa-fé do usuário, por isso, há normas delineadas para conduzir suas ações em casos de disputa sobre conteúdo, quando dois ou mais usuários estão em desacordo, ou a conduta de algum usuário apresente posturas antiéticas, violentas ou abusivas.

A definição das normas em uma wiki como essa é usualmente definida pela própria comunidade de usuários. A Wikipédia é um exemplo de sistema colaborativo (embora não empregue funcionalidades da web semântica) que possui uma vasta documentação de políticas e diretrizes para a contribuição em uma wiki (WIKIPEDIA, 2017c). Uma vez que a ela é também uma extensão do MediaWiki, suas estratégias também podem ser configuradas no Semantic MediaWiki.

Para as disputas de conteúdo, o primeiro passo é o uso do talk page, por meio do qual os usuários devem tentar alcançar um consenso. Em caso negativo, há um conjunto de ferramentas disponíveis para finalizar a disputa (SANTANA et al., 2018; WIKIPEDIA, 2017a):

a) third opinion, para requisitar a opinião de um terceiro, quando a disputa ocorre com apenas dois usuários;

b) specialised noticeboards, para requisitar a ajuda de usuários familiarizados com as políticas de conteúdo e diretrizes relevantes a um determinado mural; 
c) requests for comment, para requisitar a opinião de outros usuários não envolvidos na disputa;

d) dispute resolution noticeboard, para requisitar a moderação da disputa;

e) formal mediation, para requisitar a mediação formal ao conselho da Wiki, que irá deliberar definitivamente a respeito da disputa em andamento.

Para problemas relacionados à má conduta de usuários, o SMW oferece as seguintes ferramentas (WIKIPEDIA, 2017a):

a) sockpuppet investigations, para investigar se duas ou mais contas estão sendo operadas, de forma abusiva, pela mesma pessoa;

b) edit warring noticeboard, para reportar em um mural, o(s) usuário(s) que estão violando regras de edição;

c) administrator's noticeboards/incidentes, para requisitar ajuda de administradores e usuários experientes;

d) arbitration and arbitration enforcement, para solicitar, ao comitê, decisão relacionada a problemas contínuos de má conduta. Deverá ser acionado apenas após a falha dos demais meios de resolução de disputa.

\subsubsection{Tainacan Ontology}

O Tainacan Ontology é um sistema web de edição de ontologias de código aberto, que tem como um dos objetivos fornecer ao usuário, por meio de uma interface amigável, condições para que mesmo um leigo consiga realizar operações em uma ontologia (ACERVO CULTURA DIGITAL, 2017). Destacase que o Tainacan Ontology é um fork do Tainacan Repositório, também desenvolvido em código-aberto, utilizando o WordPress.

Dispõe de mecanismos para a participação de vários usuários na criação ou edição de uma ontologia, por meio da definição de níveis de permissões e operações específicas que o administrador pode delegar a quem desejar.

O Tainacan Ontology define um conjunto de dez tipos de entidades, as quais formam uma ontologia: classe; comentários; classificação; indivíduo; tags; atributo; valor do atributo; relação; valor da relação e metadado de termo. Para 
cada entidade é possível restringir as operações de criar, editar e apagar para quatro tipos de níveis de permissão: não permitido, anônimo, membros e aprovação (SANTANA et al., 2018).

O nível de permissão para aprovação é dividido em dois tipos, além de outras ferramentas para colaboração (SANTANA et al., 2018):

a) aprovação democrática, para aprovar por votação;

b) aprovação por moderador, para aprovar uma determinada operação por meio da autorização de um usuário moderador.

\subsubsection{WebProtégé}

O software foi baseado no editor de ontologias open source Protégé e ainda, na extensão Collaborative Protégé. Assim, o WebProtégé oferece ferramentas que permitem a criação de ontologias, mas também o trabalho colaborativo de usuários, possibilitando a comunicação, o registro de mudanças na ontologia, o controle de usuários, entre outros (TUDORACHE; VENDETTI; NOY, 2008).

No WebProtégé, a colaboração é multiusuário, em tempo real. Há definição de permissões de usuários como: 'apenas visualização', 'comentários' ou 'editor' e o compartilhamento de um projeto de ontologia com outros usuários. Existem três ferramentas básicas no WebProtégé para possibilitar a colaboração de outros (SANTANA et al., 2018; HORRIDGE et al., 2014):

a) threaded discussions, para possibilitar a comunicação entre os usuários do sistema. São marcadas como 'abertas' ou 'resolvidas', com a possibilidade de reabertura de uma discussão;

b) watches, possibilitar que usuários monitorem alterações na ontologia, por meio de notificações enviadas aos usuários via e-mail com links diretos para os termos alterados;

c) change tracking and versioning, para permitir que os usuários realizem download de versões anteriores ou revisões de uma ontologia. 


\subsubsection{Wikidata}

Assim como o SMW, o Wikidata é um projeto da Wikimedia Foundation e tem como objetivo reunir as informações de todos os demais sistemas da Wikimedia Foundation (Wikipedia, Wikimedia Commons, entre outros, inclusive o SMW) na forma de uma wiki semântica, apresentando seus dados de maneira estruturada ao usuário (WIKIPEDIA, 2017b).

As ferramentas que possibilitam a colaboratividade são variadas e abrangem situações específicas. Basicamente, existem as que tratam questões relacionadas ao conteúdo das páginas, como disputas entre usuários, informações erradas, diferença nas informações das fontes e questões relacionadas à conduta dos usuários. Assim como o SMW, o Wikidata preza por estabelecer o consenso entre usuários, mediante disputa que obedeça às diretrizes de edição de páginas e o bom comportamento dos usuários.

O Wikidata define três tipos de usuários: os $\operatorname{Admin(sysops),~que~são~}$ selecionados a partir de requisição própria, avaliada por uma comunidade, que os aprova quando há o mínimo de oito votos a favor. Aprovados, têm como função básica solucionar vandalismos, páginas em branco e outros erros, podendo restringir o acesso às páginas, apagar conteúdo e bloquear usuários; os Auto-confirmed, que são selecionados pelo software com base no número de edições realizadas no sistema, mais de 50, podendo mover páginas e editar páginas semiprotegidas e os Confirmed, que não atendendo o requisito mínimo de 50 edições, requisitam essas permissões a um administrador (SANTANA et al., 2018).

As principais ferramentas colaborativas desse sistema são:

a) project chat, para possibilitar a comunicação dos usuários a respeito de assuntos diversos;

b) request for comment, para consultar a comunidade a respeito de tópicos específicos que não puderam ser tratados/resolvidos no Project chat;

c) administrator's noticeboard/incidents, para permitir contato com os administradores do sistema a fim de discutir solicitações de mudança de nível de permissões. 

edição de ontologias por meio do Modelo $4 \mathrm{C}$ de Colaboração

Marcel Ferrante Silva, Dalton Lopes Martins, Joyce Siqueira e Douglas Veronez Santana

\subsection{Analise do Modelo 4C de Colaboração}

Considera-se como ferramenta colaborativa qualquer funcionalidade do sistema que permita ao usuário criar, alterar, remover e requisitar dados do sistema. Os quadros 1, 2 e 3 listam os nomes das ferramentas e as associam ao sistema correspondente.

Dada a possibilidade de os sistemas terem ferramentas com funcionalidades em comum, no entanto, com nomes distintos, as mesmas são listadas e associadas a um número que representa o sistema que as nomeiam. Por exemplo, no Quadro 1 há a ferramenta Commenting, associada aos números 2,3 e 6 , que correspondem aos sistemas WebProtégé, OntoWiki e MusicBrainz e a ferramenta Edit Note, associada ao número 5, que representa o sistema Tainacan Ontology. Embora tenham nomes distintos, possuem a mesma funcionalidade.

\begin{tabular}{|c|c|c|c|c|c|c|}
\hline Ferramentas para comunicação & 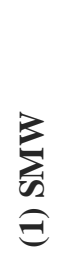 & 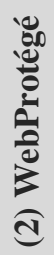 & $\underset{0}{\stackrel{0}{0}}$ & 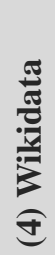 & 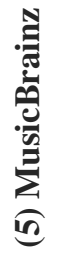 & 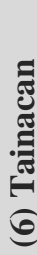 \\
\hline Talk Page & $\mathrm{x}$ & & & $\mathrm{x}$ & $\mathrm{x}$ & \\
\hline Watches & & $\mathrm{x}$ & & $\mathrm{x}$ & $\mathrm{x}$ & $\mathrm{x}$ \\
\hline Commenting $(2,3,6) /$ Edit note (5) & & $\mathrm{x}$ & $\mathrm{x}$ & & $\mathrm{x}$ & $\mathrm{x}$ \\
\hline Project chat & & & & $\mathrm{x}$ & & \\
\hline Contact the development team & & & & $\mathrm{x}$ & & \\
\hline Mailing list & & & & $\mathrm{x}$ & $\mathrm{x}$ & \\
\hline Copyright violation notice & & & & & $\mathrm{x}$ & \\
\hline Social media sharing & & & & & & $\mathrm{x}$ \\
\hline$R S S$ & & & & & & $\mathrm{x}$ \\
\hline $\begin{array}{l}\text { Contributions (4) / Collaboration } \\
\text { ranking (6) }\end{array}$ & & & & $\mathrm{x}$ & & $\mathrm{x}$ \\
\hline
\end{tabular}

Fonte: Elaborado pelos autores. 


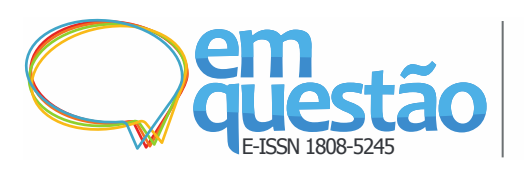

Análise dos modelos colaborativos de softwares para edição de ontologias por meio do Modelo 4C de Colaboração

Marcel Ferrante Silva, Dalton Lopes Martins, Joyce Siqueira e Douglas Veronez Santana

Quadro 2 - Ferramentas para Coordenação

\begin{tabular}{|c|c|c|c|c|c|c|}
\hline Ferramentas para coordenação & 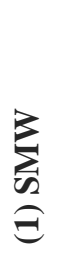 & 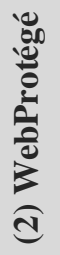 & 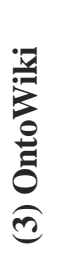 & 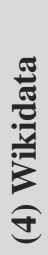 & 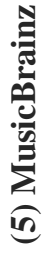 & 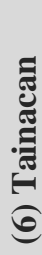 \\
\hline Conduct Code & $\mathrm{x}$ & & & & $\mathrm{x}$ & \\
\hline Arbitration and Arbitration enforcement & $\mathrm{x}$ & & & & & \\
\hline Formal mediation & $\mathrm{x}$ & & & & & \\
\hline Change tracking and versioning (2) / History (3) & & $\mathrm{x}$ & $\mathrm{x}$ & $\mathrm{x}$ & & \\
\hline Activity/provenance & & & $\mathrm{x}$ & & & \\
\hline Blocking policy & & & & $\mathrm{x}$ & & \\
\hline Page protection policy & & & & $\mathrm{x}$ & & \\
\hline Style guidelines & & & & & $\mathrm{x}$ & \\
\hline Moderator approval & $\mathrm{x}$ & & & $\mathrm{X}$ & $\mathrm{x}$ & $\mathrm{x}$ \\
\hline Permission configuration for collection creation & & & & & & $\mathrm{x}$ \\
\hline Permission configuration for collection deletion & & & & & & $\mathrm{x}$ \\
\hline Create user & & & & & & $\mathrm{x}$ \\
\hline Edit user data & & & & & & $\mathrm{x}$ \\
\hline Delete user registry & & & & & & $\mathrm{x}$ \\
\hline
\end{tabular}

Fonte: Elaborado pelos autores.

Quadro 3 - Ferramentas para Colaboração e Cooperação

\begin{tabular}{|c|c|c|c|c|c|c|c|c|c|c|c|c|c|}
\hline $\begin{array}{l}\text { Ferramentas para } \\
\text { Colaboração e } \\
\text { Cooperação }\end{array}$ & $\sum_{\infty}^{B}$ & 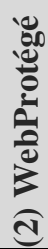 & 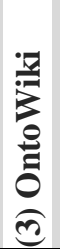 & 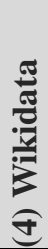 & 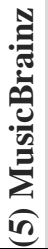 & 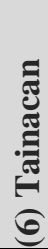 & $\begin{array}{l}\text { Ferramentas } \\
\text { para } \\
\text { Colaboração e } \\
\text { Cooperação }\end{array}$ & 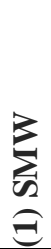 & 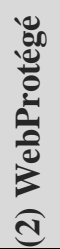 & مْ & 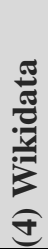 & 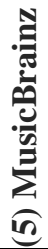 & 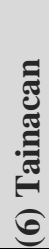 \\
\hline Create page & $\mathrm{x}$ & & & $\mathrm{x}$ & & & $\begin{array}{l}\text { Request a } \\
\text { query }\end{array}$ & & & & $\mathrm{X}$ & & \\
\hline Add property & $\mathrm{x}$ & & & $\mathrm{x}$ & & & $\begin{array}{l}\text { Request for } \\
\text { permissions }\end{array}$ & & & & $\mathrm{x}$ & & \\
\hline Create category & $\mathrm{x}$ & & & $\mathrm{x}$ & & & Add Artist & & & & & $\mathrm{x}$ & \\
\hline $\begin{array}{l}\text { Request for } \\
\text { comments }\end{array}$ & $\mathrm{x}$ & & & $\mathrm{x}$ & & & Add Release & & & & & $\mathrm{X}$ & \\
\hline $\begin{array}{l}\text { Specialised } \\
\text { noticeboards }\end{array}$ & $\mathrm{x}$ & & & & & & $\begin{array}{l}\text { Add } \\
\text { Standalone } \\
\text { Recording }\end{array}$ & & & & & $\mathrm{X}$ & \\
\hline $\begin{array}{l}\text { Dispute resolution } \\
\text { noticeboards }\end{array}$ & $\mathrm{x}$ & & & & & & Add Work & & & & & $\mathrm{X}$ & \\
\hline Third opinion & $\mathrm{x}$ & & & & & & Add Place & & & & & $\mathrm{x}$ & \\
\hline $\begin{array}{l}\text { Administrator's } \\
\text { noticeboard }\end{array}$ & $\mathrm{x}$ & & & $\mathrm{x}$ & & & Add Event & & & & & $\mathrm{x}$ & \\
\hline $\begin{array}{l}\text { Sockpuppet } \\
\text { investigations }\end{array}$ & $\mathrm{x}$ & & & & & & $\begin{array}{l}\text { Add a } \\
\text { Relationship }\end{array}$ & & & & & $\mathrm{X}$ & \\
\hline $\begin{array}{l}\text { Threaded } \\
\text { discussions }\end{array}$ & & $\mathrm{x}$ & & & & & Votting & & & & & $\mathrm{x}$ & $\mathrm{x}$ \\
\hline Ontology sharing & & $\mathrm{x}$ & & & & $\mathrm{X}$ & $\begin{array}{l}\text { Request Item } \\
\text { Creation }\end{array}$ & & & & $\mathrm{X}$ & & $\mathrm{x}$ \\
\hline
\end{tabular}




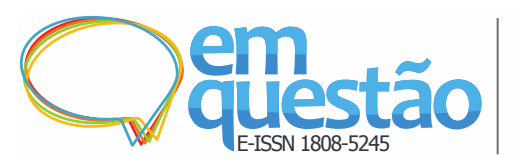

Análise dos modelos colaborativos de softwares para edição de ontologias por meio do Modelo $4 \mathrm{C}$ de Colaboração

Marcel Ferrante Silva, Dalton Lopes Martins, Joyce Siqueira e Douglas Veronez Santana

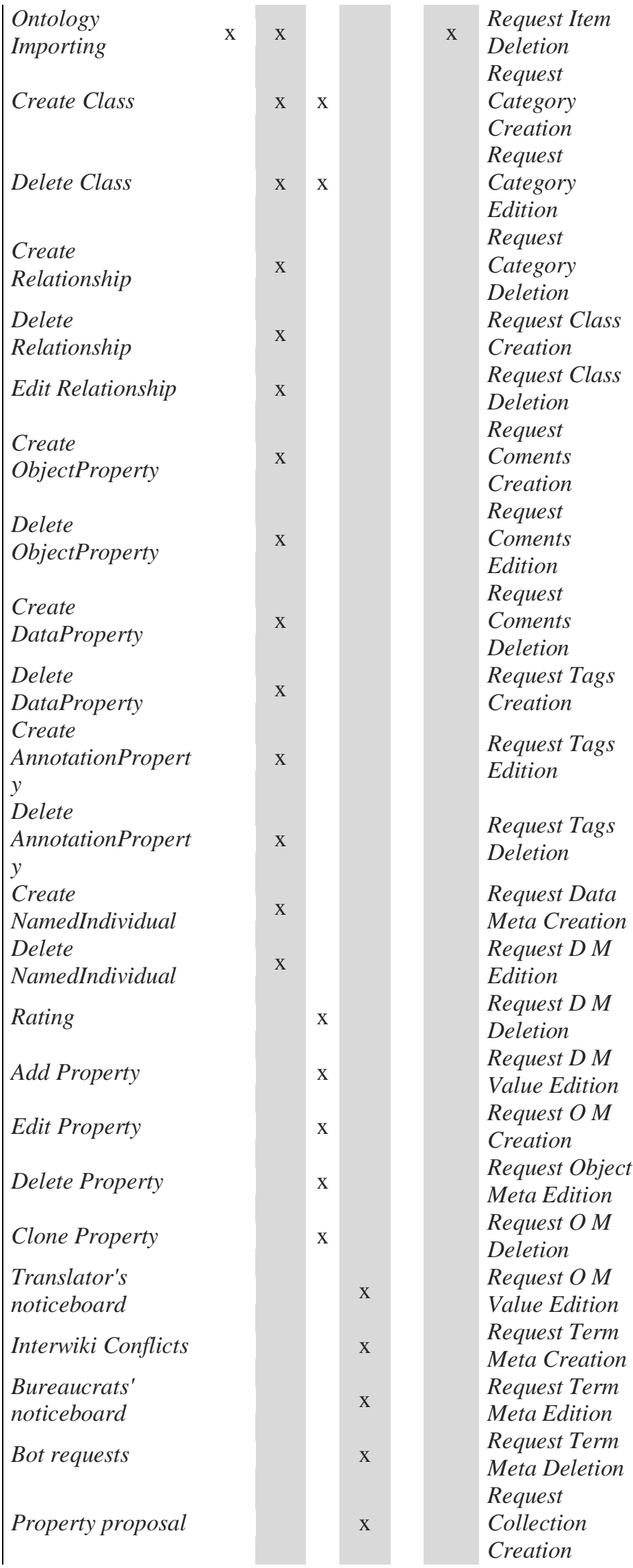

$\mathrm{X}$

$\mathrm{x}$

$\mathrm{x}$

$\mathrm{X}$

$\mathrm{x}$

$\mathrm{X}$

$\mathrm{X}$

$\mathrm{x}$

$\mathrm{x}$

$\mathrm{X}$

$\mathrm{X}$

$\mathrm{x}$

$\mathrm{x}$

$\mathrm{X}$

$\mathrm{X}$

$\mathrm{X}$

$\mathrm{x}$

$\mathrm{X}$

$\mathrm{X}$

$\mathrm{X}$

$\mathrm{X}$

$\mathrm{X}$

$\mathrm{X}$

$\mathrm{X}$ 

edição de ontologias por meio do Modelo 4C de Colaboração

Marcel Ferrante Silva, Dalton Lopes Martins, Joyce Siqueira e Douglas Veronez Santana

\begin{tabular}{|c|c|c|c|}
\hline $\begin{array}{l}\text { Properties for } \\
\text { deletion }\end{array}$ & $\mathrm{X}$ & $\begin{array}{l}\text { Request } \\
\text { Collection } \\
\text { Deletion }\end{array}$ & $\mathrm{X}$ \\
\hline
\end{tabular}

Fonte: Elaborado pelos autores.

A Tabela 1 apresenta o resultado considerando a quantidade de itens avaliados com a quantidade de itens encontrados.

Tabela 1 - Quantidade de ferramentas colaborativas identificadas nos sistemas para edição de ontologias em comparação com o número total de ferramentas avaliadas

\begin{tabular}{|c|c|c|c|c|c|c|c|c|c|c|c|c|c|}
\hline & & & & $\begin{array}{r}\text { W } \\
\text { Pro }\end{array}$ & $\begin{array}{l}\text { eb } \\
\text { tégé }\end{array}$ & Ont & Wiki & Wik & & Musi & Brainz & Tain & Iacan \\
\hline & & Qnt & $\begin{array}{c}\text { Perc } \\
\%\end{array}$ & Qnt & $\begin{array}{c}\text { Perc } \\
\%\end{array}$ & Qnt & $\begin{array}{c}\text { Perc } \\
\%\end{array}$ & Qnt & $\begin{array}{c}\text { Perc } \\
\%\end{array}$ & Qnt & $\begin{array}{c}\text { Perc } \\
\%\end{array}$ & Qnt & $\begin{array}{c}\text { Perc } \\
\%\end{array}$ \\
\hline Comu & 10 & 1 & 10,0 & 2 & 20,0 & 1 & 10,0 & 6 & 60,0 & 5 & 50,0 & 5 & 50,0 \\
\hline $\begin{array}{l}\text { Colabc } \\
\text { e Coop }\end{array}$ & 73 & 10 & 13,7 & 16 & 21,9 & 7 & 9,6 & 14 & 19,2 & 8 & 11,0 & 29 & 39,7 \\
\hline Coordenação & 14 & 4 & 28,6 & 1 & 7,1 & 2 & 14,3 & 4 & 28,6 & 3 & 21,4 & 6 & 42,9 \\
\hline Totais & 97 & 15 & 15,5 & 19 & 19,6 & 10 & 10,3 & 24 & 24,7 & 16 & 16,5 & 40 & 41,2 \\
\hline
\end{tabular}

O resultado apresenta o Tainacan Ontology como o software que contempla o maior número de itens avaliados, atingindo 41,2\%. Além disso, pode-se perceber que os segundo e terceiro colocados, Wikidata e WebProtégé $(24,7 \%$ e $19,6 \%)$ possuem uma diferença considerável em relação ao primeiro. Ainda assim, fica nítido o quanto todas as ferramentas podem evoluir na implementação de novos recursos.

Dando continuidade à análise, a Tabela 2 mostra o resultado da contagem do número de ferramentas colaborativas de cada sistema, classificadas em relação a cada um dos C's do Modelo 4C. Essa análise permite identificar qual o maior foco dos sistemas para edição de ontologias.

Ao analisar os sistemas, apesar da grande diferença em número de ferramentas, a verificação da porcentagem em relação ao total de ferramentas colaborativas que os sistemas oferecem ajuda a identificar a expressividade das mesmas em cada sistema. 
Marcel Ferrante Silva, Dalton Lopes Martins, Joyce Siqueira e Douglas Veronez Santana

Tabela 2- Quantidade de ferramentas colaborativas identificadas nos sistemas para edição de ontologias, classificadas de acordo com o C's do Modelo 4C

\begin{tabular}{|c|c|c|c|c|c|c|c|c|c|c|c|c|}
\hline \multirow[b]{2}{*}{ Modelo 4C } & \multicolumn{2}{|c|}{ SMW } & \multicolumn{2}{|c|}{ WebProtégé } & \multicolumn{2}{|c|}{ OntoWiki } & \multicolumn{2}{|c|}{ Wikidata } & \multicolumn{2}{|c|}{ MusicBrainz } & \multicolumn{2}{|c|}{ Tainacan } \\
\hline & Qnt & $\begin{array}{c}\text { Perc } \\
\%\end{array}$ & Qnt & $\begin{array}{c}\text { Perc } \\
\%\end{array}$ & Qnt & $\begin{array}{c}\text { Perc } \\
\%\end{array}$ & Qnt & $\begin{array}{c}\text { Perc } \\
\%\end{array}$ & Qnt & $\begin{array}{c}\text { Perc } \\
\%\end{array}$ & Qnt & $\begin{array}{c}\text { Perc } \\
\%\end{array}$ \\
\hline Comunicação & 1 & 6,7 & 2 & 10,5 & 1 & 10,0 & 6 & 25,0 & 5 & 31,2 & 5 & 12,5 \\
\hline $\begin{array}{l}\text { Colaboração } \\
\text { e Cooperação }\end{array}$ & 10 & 66,7 & 16 & 84,2 & 7 & 70,0 & 14 & 58,3 & 8 & 50,0 & 29 & 72,5 \\
\hline Coordenação & 4 & 26,7 & 1 & 5,3 & 2 & 20,0 & 4 & 16,7 & 3 & 18,7 & 6 & 15,0 \\
\hline Totais & 15 & 100,0 & 19 & 100,0 & 10 & 100,0 & 24 & 100,0 & 16 & 100,0 & 40 & 100,0 \\
\hline
\end{tabular}

Observa-se na Tabela 2 que os números totais de ferramentas colaborativas diferem muito entre si. Embora os sistemas SMW, WebProtégé, OntoWiki e MusicBrainz tenham uma quantidade similar de ferramentas (15, 19, 10 e 16, respectivamente), os sistemas Wikidata e, principalmente, o Tainacan Ontology, apresentam maior variação em relação aos demais (24 e 40, respectivamente).

Analisando o aspecto da Comunicação e observando os sistemas que mais se destacam nesse aspecto, tem-se que o WebProtégé, o MusicBrainz e o Tainacan Ontology se destacam em número de ferramentas (6, 5 e 5, respectivamente). É importante observar também a porcentagem das ferramentas para Comunicação de cada sistema, pois, embora os sistemas MusicBrainz e Tainacan Ontology possuam o mesmo número de ferramentas, as ferramentas do MusicBrainz representam $31,25 \%$ enquanto as do Tainacan Ontology representam $12,5 \%$ do total. Ao mesmo tempo, tem-se que o Wikidata dedica em torno de $25 \%$ de suas ferramentas para a Comunicação. Dessa forma, no aspecto Comunicação, o MusicBrainz se destaca em comparação aos demais.

Sob a ótica da Colaboração e Cooperação, todos os sistemas analisados neste artigo dedicam mais da metade de suas ferramentas colaborativas para possibilitar a colaboração e a cooperação entre os usuários. Destacam-se, nesse aspecto, os sistemas WebProtégé e o Tainacan Ontology. O WebProtégé dedica mais de $84 \%$ de suas ferramentas para a colaboração e o Tainacan Ontology mais de $72 \%$. Apesar disso, há certa diferença no número de ferramentas que 
cada sistema dedica para a Colaboração e Cooperação: o WebProtégé dedica 16 ferramentas, enquanto o Tainacan Ontology, 29.

Referente ao aspecto de Coordenação observa-se que, embora os sistemas SMW e Wikidata possuam o mesmo número de ferramentas, 4, o primeiro dedica em torno de $10 \%$ a mais que o último a esse aspecto. Por outro lado, o Tainacan Ontology, com o maior número de ferramentas, 6, contempla $15 \%$ do seu total de ferramentas. O sistema OntoWiki, que dedica a maior porcentagem, 20\%, a esse aspecto, se comparado aos demais, equivale a apenas 2 ferramentas do seu total.

Na Figura 1, gerada a partir da Tabela 2, podemos analisar cada sistema individualmente, verificando qual dos aspectos do Modelo 4C mais se destaca em cada um. É notório que todos os sistemas analisados dedicam grande parte de suas ferramentas colaborativas para a Colaboração e Cooperação.

Figura 1 - Número de ferramentas colaborativas classificadas segundo o Modelo 4C de colaboração.

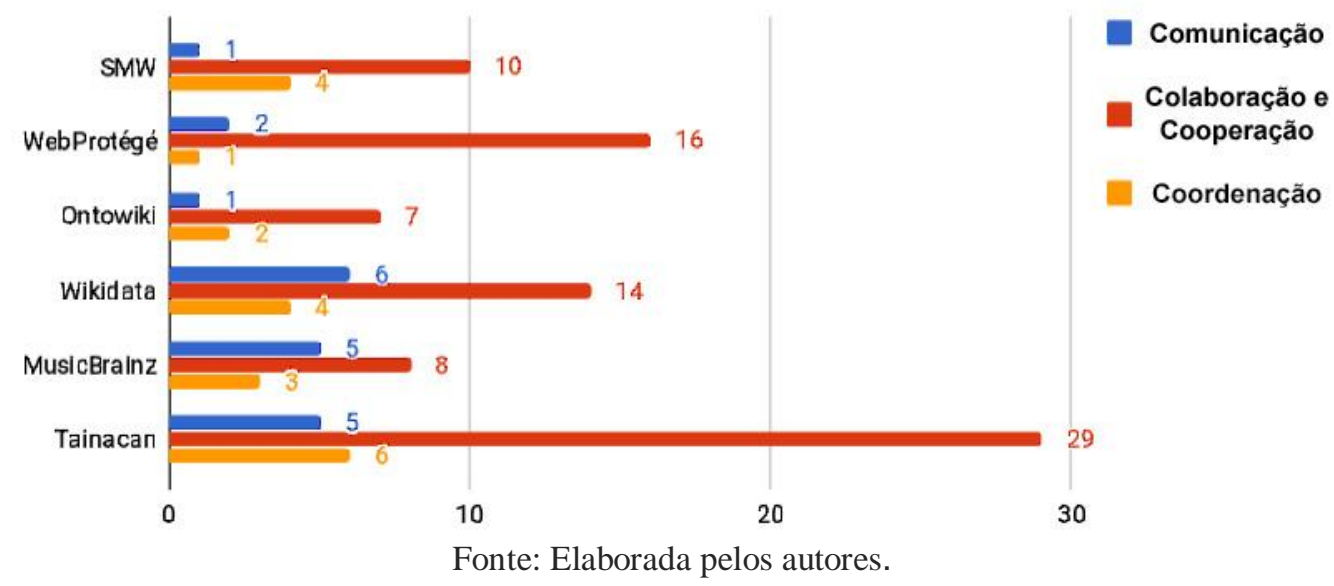

Pode-se observar que o Tainacan Ontology é o que mais se destaca, com 29 ferramentas. Os demais, com exceção do MusicBrainz e do OntoWiki, oferecem entre 10 e 16 ferramentas para Colaboração e Cooperação. Outra informação importante é que a Comunicação é o segundo aspecto colaborativo mais visado pelos sistemas, com exceção do SMW e do OntoWiki.

A Figura 2, também gerada a partir da Tabela 2, apresenta a porcentagem que cada sistema dedicou para a Comunicação, Colaboração e Cooperação e Coordenação. 
Figura 2 - Porcentagem do total de ferramentas colaborativas de cada sistema dedicadas para cada aspecto do Modelo 4C de Colaboração

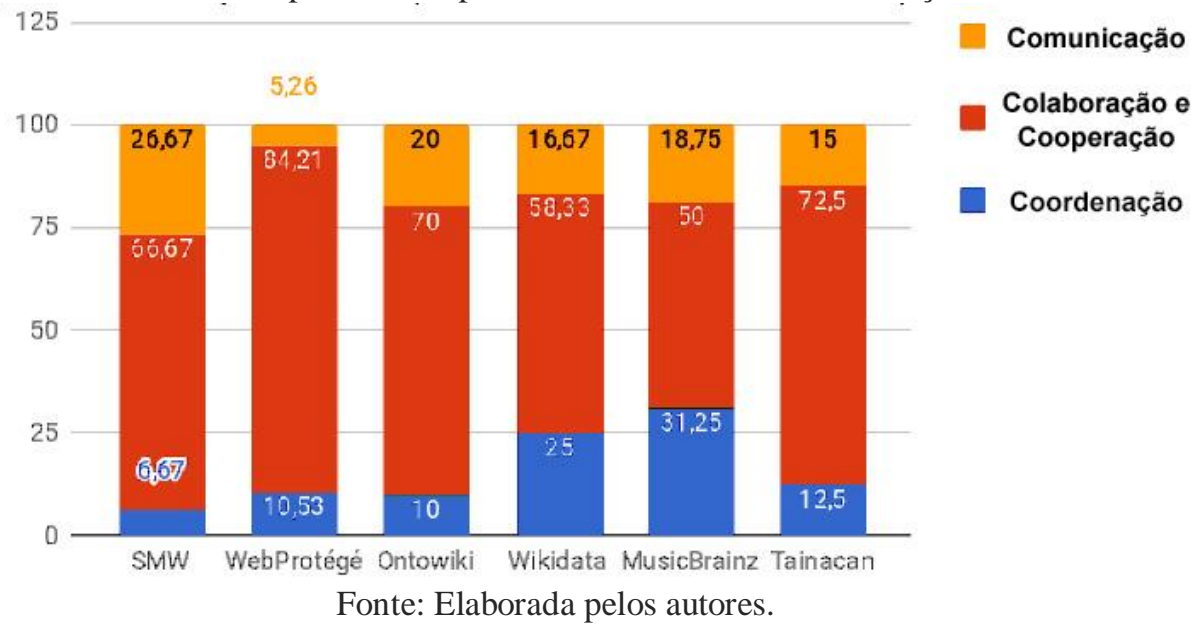

Fica nítido na Figura 2, a importância dada às ferramentas que possibilitam a Colaboração e Cooperação, destacando-se o sistema WebProtégé, que dedicou mais de $84 \%$ do total de suas ferramentas colaborativas para a Colaboração e Cooperação. Os demais sistemas dedicaram entre 50\% e 75\%, com destaque para os sistemas SMW, OntoWiki e Tainacan Ontology.

Analisando as ferramentas de cada sistema (vide Tabela 2), nota-se que os três sistemas que mais se destacam em Colaboração e Cooperação dispõem de ferramentas que lidam diretamente com as entidades OWL. Esses sistemas permitem que o usuário realize operações (adicionar, editar, remover) sobre indivíduos, propriedades (ObjectProperties, DataProperties), relacionamentos, Annotation Properties, entre outros, de modo que, quanto maior o número de entidades que cada sistema dispõe, maior o número de ferramentas que possibilitam a Colaboração e Cooperação.

É importante ressaltar, entretanto, que cada sistema, em especial os que mais se destacaram em Colaboração e Cooperação, lida com as entidades OWL de maneira distinta. Enquanto alguns modelam suas entidades exatamente igual à nomenclatura e especificação das entidades OWL (ObjectPropertie, DataPropertie, AnnotationPropertie), como no caso do WebProtégé, alguns utilizam outras abordagens para suas entidades, generalizando os nomes (de ObjectPropertie, DataPropertie e AnnotationPropertie para apenas Propertie, 
por exemplo), como no caso do sistema OntoWiki. Como o número de entidades é reduzido, o número de ferramentas colaborativas também.

O Tainacan Ontology e o SMW utilizam sua própria abordagem para modelar as entidades OWL: o Tainacan Ontology modela as entidades OWL atribuindo nomes distintos (metadado de dado, metadado de termo, categoria, coleção etc.), conferindo ao usuário a capacidade de poder utilizar os recursos sem conhecer sua nomenclatura. No caso dos sistemas SWM e Wikidata, embora possuam $66,67 \%$ e 58,33\%, respectivamente, de suas ferramentas dedicadas à Colaboração e Cooperação, boa parte de suas ferramentas tem como objetivo resolver disputas de conteúdo e resolver problemas de conduta de usuários, como mostra a Tabela 2.

Assim, dentre os sistemas analisados observou-se, como citado anteriormente, um foco notável para ferramentas colaborativas que permitem a Colaboração e Cooperação. O SMW e o Wikidata adequam suas ferramentas para um contexto de cooperação no ambiente de uma wiki, dispondo de uma maioria de ferramentas que tem por objetivo resolver disputas de conteúdo e de conduta entre os usuários. O SMW se diferencia do Wikidata no que se refere ao tratamento dado às entidades que permitem a modelagem da informação como uma ontologia. Ambos os sistemas são de autoria da Wikimedia Foundation e o Wikidata é um aprimoramento em termos de mecanismos para modelagem da informação como ontologia (propriedades e entidades OWL).

O MusicBrainz, desenvolvido com um foco muito específico, a área musical, é notável pela possibilidade de participação da comunidade de usuários por meio da votação. O sistema OntoWiki, comparado aos sistemas WebProtégé e Tainacan Ontology, oferece um número menor de entidades, impactando diretamente no número de ferramentas colaborativas. Por fim, os sistemas WebProtégé e Tainacan Ontology são os que mais se destacam nas ferramentas colaborativas referentes à cooperação, com a diferença de que o WebProtégé nomeia suas entidades de forma similar à especificação da linguagem OWL, o que é vantajoso para quem já conhece a linguagem, e o Tainacan Ontology nomeia suas entidades de modo a permitir que um usuário leigo possa criar ontologias. Outra característica marcante do Tainacan Ontology é a 
possibilidade de definir aprovação por moderação e aprovação democrática, por meio de votação.

\section{Considerações finais}

A colaboração entre os membros de um sistema de edição de ontologias é de suma importância para disponibilização de ontologias confiáveis e ricas. A partir dos resultados dessa pesquisa, constatou-se que todos os sistemas para edição de ontologias supracitados oferecem ferramentas que possibilitam essa colaboração.

Além disso, por meio da avaliação dos softwares, foi possível identificar em qual dos 4 C's cada sistema se destaca mais, se na Comunicação, na Colaboração e Cooperação ou na Coordenação. Em suma, obteve-se que: considerando a quantidade de itens avaliados, que totalizaram 97 itens, destacase o Tainacan Ontology (40), seguido do Wikidata (24) e demais softwares. Em se tratando especificamente sobre a Comunicação, constata-se que esta não é o foco dos softwares, que dedicam poucas de suas funcionalidades a este quesito. Ainda assim, os que se destacam são o Wikidata (6), seguido pelo MusicBrainz (5) e Tainacan Ontology (5), empatados em segundo lugar. Por outro lado, a Colaboração e a Cooperação, são vistas como principal foco dos softwares editores de ontologias. O Tainacan Ontology possui o maior número de funcionalidades (26), seguido do WebProtégé (16) e demais softwares. Na Coordenação, o Tainacan Ontology (6) também se destaca em relação aos demais, seguido do SMW (4) e Wikidata (4), empatados na segunda posição.

Conclui-se que a metodologia utilizada é eficiente e gerou resultados importantes em relação aos softwares editores de ontologias. Apresentou pontos fortes e pontos de atenção, que podem colaborar com futuras implementações nos projetos, ou até mesmo, subsidiar novos softwares.

\section{Referências}

ACERVO CULTURA DIGITAL. Tainacan manual do usuário. 2017. 
AUER, Sören; DIETZOLD, Sebastian; RIECHERT, Thomas. OntoWiki - a tool for social, semantic collaboration. In: CRUZ, Isabel et al. (Ed.) The semantic web - ISWC 2006. Berlin: Springer, 2006. p. 736-749. (Lecture Notes in Computer Science, 4273)

BASSANI, Patricia. B. Scherer.; REIS, Adriana dos; DALANHOL, Daniel. Análise da colaboração em ambientes digitais para compartilhamento de atividades de aprendizagem: uma perspectiva com base em Learning Design. In: BRAZILIAN SYMPOSIUM ON COMPUTERS IN EDUCATION, 27., Uberlândia, p. 1215, 2016. Proceedings... Uberlândia: Universidade Federal de Uberlândia, 2016.

CASTRO, Francisco Gómez, GONÇALVES, Berenice Santos; FIGUEIREDO, Luiz Fernando. Mapeamento de características de sites de compartilhamento de refeições baseado no Modelo 3C de Colaboração. Navus: revista de gestão e tecnologia, Florianópolis, v. 7, n. 1, p. 29-42, 2017.

COSTA, António Pedro et al. Análise de interações focada na colaboração e cooperação do modelo 4C. Revista Lusófona de Educação, Lisboa, v. 29, n. 29, jun. 2015.

COSTA, António Pedro; LOUREIRO, Maria João; REIS, Luis Paulo. Do modelo 3C de colaboração ao modelo 4C: modelo de análise de processos de desenvolvimento de software educativo. Revista Lusófona de Educação, Lisboa, v. 27, n. 27, p. 181-200, nov. 2014.

COSTA, Antóno Pedro; REIS, Luis Paulo; LOUREIRO, Maria João.

Desenvolvimento de software educativo: a coordenação como fator crítico de sucesso. Revista Ibérica de Sistemas e Tecnologias de Informação, Rio Tinto, n. E2, p. 1-18, 2014.

CRUZ JÚNIOR, Geraldo Gomes da et al. Um estudo para implantação de uma rede social à luz do modelo $3 \mathrm{C}$ e das ontologias de colaboração: o caso CirCor. In: SIMPÓSIO BRASILEIRO DE SISTEMAS COLABORATIVOS, 12., Salvador. Anais... Salvador: UFBA, 2015.

FUKS, Hugo et al. Applying the 3C Model to Groupware Development. International Journal of Cooperative Information Systems, Cingapura, v. 14, n. 2-3, p.299-328, 2005.

GRUBER, Thomas R. Toward Principles for the Design of Ontologies Used for Knowledge sharing. International Journal Human-Computer Studies, Padova, n. 43. p. 907-928, nov. 1995.

HORRIDGE, Matthew et al. WebProtégé: a collaborative Web-based platform for editing biomedical ontologies. Bioinformatics, Oxford, v. 30, n. 16, p. 2384- 
2385, ago. 2014.

MELO, Áurea Hiléia da Silva et al. Modelo 3C de Colaboração aplicado ao uso de um repositório para o desenvolvimento de interfaces para autistas. In: SIMPÓSIO BRASILEIRO DE SISTEMAS COLABORATIVOS, 14., 2017, São Paulo. Anais... São Paulo: Sociedade Brasileira de Computação, 2017. p. 1471-1485.

MUSICBRAINZ. Introduction to editing. 2017a. Disponível em: <https://musicbrainz.org/doc/Introduction_to_Editing>. Acesso em $14 \mathrm{dez}$. 2017.

MUSICBRAINZ. Introduction to voting. 2017b. Disponível em: <https://musicbrainz.org/doc/Introduction_to_Voting>. Acesso em 14 dez. 2017.

MUSICBRAINZ. MusicBrainz documentation. 2017c. Disponível em: <https://musicbrainz.org/doc/MusicBrainz_Documentation>. Acesso em $14 \mathrm{dez}$. 2017.

OLIVEIRA, Eduarda Bodaneze de; DUTRA, Moisés Lima. Um levantamento sobre do uso de ferramentas da Web 2.0 entre os estudantes da Ciência da Informação da Universidade Federal de Santa Catarina. Encontros Bibli, Florianópolis, v. 19, n. 39, p. 153-182, jan./abr. 2014.

PROTÉGÉ. WebProtégé user guide. 2015. Disponível em:

<https://protegewiki.stanford.edu/wiki/WebProtegeUsersGuide>. Acesso em: 14 dez. 2017.

SANTANA, Douglas Veronez et al. Avaliação de modelos para colaboração em softwares editores de ontologia. Revista Brasileira de Biblioteconomia e Documentação, São Paulo, v. 14, n. 3, p. 123-141, set. 2018.

SEMANTIC MEDIAWIKI. General - Semantic MediaWiki. 2017. Disponível em: <https://www.semanticmediawiki.org/wiki/Semantic_MediaWiki>. Acesso em: 13 nov. 2017.

SEVERINO, A. J. Metodologia do trabalho científico. Perdizes: Cortez editora, 2017.

TAINACAN.ORG. Tainacan manual do usuário. 2017. Disponível em: <http://tainacan.org/wp-content/uploads/2017/02/Manual-Repositorio.pdf >. Acesso em: 14 dez. 2017.

TUDORACHE, Tania; VENDETTI, Jennifer; NOY, Natalya Fridman. WebProtégé: a lightweight OWL ontology editor for the web. In:

INTERNATIONAL WORKSHOP OWL: EXPERIENCES AND 
DIRECTIONS, 5., 2008, Karlsruhe. Proceedings... Karlsruhe:

Kongresszentrum, 2008.

VITAL, Luciane Paula; CAFÉ, Ligia Maria Arruda. Ontologias e taxonomias: diferenças. Perspectivas em Ciência da Informação, Belo Horizonte, v. 16, n. 2, p. 115-130, abr. 2011.

WIKIDATA. Main page. 2018. Disponível em:

<https://www.wikidata.org/wiki/Wikidata:Main_Page>. Acesso em: 16 set 2018.

WIKIPEDIA. Dispute resolution requests. 2017a. Disponível em: <https://en.wikipedia.org/wiki/Wikipedia:Dispute_resolution_requests >. Acesso em: 11 dez. 2017.

WIKIPEDIA. Wikidata. 2017b. Disponível em: <https://en.wikipedia.org/wiki/Wikidata >. Acesso em $11 \mathrm{dez} 2017$.

WIKIPEDIA. Wikipedia: policies and guidelines. 2017c. Disponível em: <https://en.wikipedia.org/wiki/Wikipedia:Policies_and_guidelines >. Acesso em: 19 dez. 2017.

\title{
Analysis of the collaborative models of systems for ontology editing through the Collaboration Model 4C
}

\begin{abstract}
This research aims to present the collaboration models of six ontology software publishers: MusicBrainz, OntoWiki, Semantic MediaWiki, Tainacan Ontology, WebProtégé and Wikidata, and to check the available tools by classifying them according to the Collaboration Model $4 \mathrm{C}$, which covers communication, coordination and collaboration and cooperation. The result covers several nuances about the software and its participation in each ' $\mathrm{C}$ ' of the Model, but, in general, it presents the WebProtégé with the highest number of communication requirements, while Tainacan has the highest number of requirements related to coordination and cooperation. The software collaboration model is essential to promote interaction, in order to mediate the divergences and to enable effective collaboration, therefore the more complete the better the service offered so it is expected that these results will serve as a basis for updating the existing software or for creating new ones.
\end{abstract}

Keywords: 4C Collaboration Model. Software Ontology Editors. Collaboration Model.

Rececido : 21/12/2017

Aceito: 19/06/2018 


\section{APÊNDICE A - Definição das ferramentas colaborativas dos softwares editores de ontologias}

As ferramentas colaborativas encontradas nos softwares têm suas funções detalhadas a seguir (MUSICBRAINZ, 2017c), (WIKIDATA, 2018), (TAINACAN.ORG, 2017) e (PROTÉGÉ, 2015).

\section{A.1. Comunicação}

○ Talk Pages: oferece um meio para realização de discussões referente a melhorias ou divergências quanto a conteúdo.

○ Watches: permite monitorar mudanças na ontologia. Sempre que atualizadas, o sistema notificará o usuário via e-mail, com links diretos ao termo editado.

- Commenting / Edit note: permite a inclusão de comentários ou observações sucintas a respeito de um determinado item, por meio de caixas de texto, como em um formulário.

○ Project chat: oferece um meio para realização de discussões variadas, tais como políticas e propostas de operação do sistema, itens de dados específicos, questões técnicas, etc.

- Contact the development team: possibilita o contato com a equipe de desenvolvimento do sistema, seja para propor sugestões ou reportar bugs.

○ Mailing list: lista de e-mail que oferece suporte para comunicação sobre assuntos relacionados ao projeto do sistema para edição de ontologias, assim como para reportar bugs.

- Copyright violation notice: permite que o usuário notifique os administradores, por e-mail, sobre qualquer tipo de violação de direitos autorais.

○ Social media sharing: permite aos usuários compartilhar conteúdo do sistema em redes sociais.

○ RSS: permite ao usuário obter um link utilizado por meio de um leitor RSS, noticiando-o sobre atividades ocorridas no sistema. 
- Contribution / Collaboration ranking: exibe uma listagem, relacionando os usuários que mais contribuíram sobre um determinado conteúdo.

\section{A.2. Colaboração e Cooperação}

○ Create page: permite criar uma página web destinada à sua nova contribuição no sistema.

- Add property: permite adicionar uma nova propriedade. Nesse caso, uma propriedade pode estabelecer uma relação entre indivíduos, assim como atribuir tipagem ao atributo de um indivíduo.

- Create category: permite classificar indivíduos, categorizando-os.

- Request for comments: permite solicitar a opinião de terceiros a respeito de assuntos variados. É uma maneira de atrair atenção de outros usuários às alterações em páginas, políticas do sistema, orientações, etc. Usa um sistema de painéis de informação centralizados e aleatórios e convites entregues por bots. Um bot é, essencialmente, um programa que automatiza tarefas como criação de itens, adição, criação ou importação de declarações em itens, entre outras coisas.

- Specialised noticeboards: permite realizar perguntas e solicitar ajuda a usuários familiarizados com políticas de conteúdo e diretrizes relacionadas a um assunto específico.

- Dispute resolution noticeboards: permite solicitar moderação a respeito de um assunto no qual não foi possível chegar a um consenso por meio da página de discussão (Talk page).

- Third opinion: requisita uma opinião externa quando há uma disputa de conteúdo entre dois usuários.

- Administrator's noticeboard / incidents: requisita assistência de um administrador ou de um usuário experiente a respeito da má conduta de um usuário.

○ Sockpuppet investigations: permite abrir uma investigação para verificar se duas ou mais contas estão sendo abusivamente utilizadas pelo mesmo usuário. 
- Threaded discussions: permite exibir, em um contêiner, os comentários dos usuários relacionados a uma determinada entidade de uma ontologia.

- Ontology sharing: permite compartilhar uma ontologia com outros usuários do sistema.

○ Ontology importing: permite importar uma ontologia para o sistema atual.

- Create/Delete class: permite criar ou apagar uma classe. De acordo com a linguagem OWL, classe pode ser entendido como um conjunto de indivíduos.

- Create/Edit/Delete relationship: permite criar, editar ou remover relacionamentos entre indivíduos e propriedades de uma ontologia.

○ Create/Delete objectProperty: permite criar ou remover um relacionamento entre propriedades.

- Create/Delete dataProperty: permite criar ou remover uma propriedade que recebe entrada de texto por parte do usuário.

- Create/Delete annotation: permite criar ou apagar uma anotação relacionada a alguma entidade ou axioma da ontologia.

- Create/Delete namedIndividual: permite criar ou remover um indivíduo de uma coleção.

○ Ratting: permite avaliar, por meio de uma nota, um determinado item de uma ontologia.

○ Add/Edit/Delete/Clone property: permite adicionar, editar, remover ou clonar uma propriedade de uma determinada ontologia.

○ Translators' noticeboard: permite a interação de usuários que colaboram na tradução de conteúdo do sistema.

○ Interwiki Conflicts: permite relatar discordâncias entre um item do Wikidata e outras páginas de algum outro sistema da Wikimedia. Como o Wikidata propõe a unificação das várias bases de dados mantidas pela Wikimedia Foundation, essa é uma importante ferramenta colaborativa.

- Bureaucrats' noticeboard: permite consultar um administrador do tipo bureaucrat sobre assuntos dos quais o mesmo é responsável por monitorar. 
○ Bot requests: permite requisitar a operação de um bot sobre uma entidade ou conjunto de entidades. O usuário justifica exatamente o motivo de sua requisição, podendo ser atendida ou não.

○ Property proposal: permite propor a criação de uma propriedade possibilitando discussão entre vários usuários sobre a validade e viabilidade de tal operação.

- Properties for deletion: permite propor a exclusão de uma determinada propriedade, possibilitando discussão entre vários usuários sobre a validade e viabilidade de tal operação.

○ Request a query: permite realizar a solicitação de consultas na linguagem SPARQL.

○ Request for deletions: permite solicitar a remoção de um determinado item do sistema. Os critérios de remoção variam de acordo com algumas políticas ou práticas não permitidas, como por exemplo, o vandalismo.

- Request for permissions: permite solicitar aos administradores a alteração do nível de permissão atual.

- Add artist: permite cadastrar informações de um artista.

- Add release: permite cadastrar informações a respeito de um álbum ou single.

- Add standalone recording: permite cadastrar uma gravação (música, por exemplo) pertencente a um determinado álbum.

- Add work: permite adicionar uma criação original artística ou intelectual do artista. Pode ser, além de uma música, um poema, uma novela, etc.

- Add place: permite adicionar informações a respeito do lugar onde a música foi gravada/apresentada.

- Add event: permite adicionar informações a respeito de apresentações, festivais, concertos, entre outros.

- Add relationship: permite estabelecer relacionamentos entre as entidades work, standalone recording, place, event e artist.

○ Votting: permite votar, com o intuito de aprovar ou rejeitar uma determinada alteração em alguma entidade do sistema. 
○ Request item creation/deletion: permite requerer a criação ou remoção de um item.

- Request category creation/edition/deletion: permite requerer a criação, edição ou remoção de uma categoria.

- Request classification creation/deletion: permite requerer a criação ou remoção de uma classificação.

- Request comments creation/edition/deletion: permite requerer a criação, edição ou remoção de um comentário.

- Request tags creation/edition/deletion: permite requerer a criação/edição/remoção de uma tag.

○ Request data metadata creation/edition/deletion: permite requerer a criação, edição ou remoção de um metadado de dado.

○ Request object metadata creation/edition/deletion: permite requerer a criação, edição ou remoção de um metadado de objeto.

- Request object metadata value edition: permite requerer a edição do valor de um metadado de objeto.

○ Request term metadata creation/edition/deletion: permite requerer a criação, edição ou remoção de um metadado de termo.

○ Request collection creation/deletion: permite requerer a criação ou remoção de uma coleção.

\section{A.3. Coordenação}

○ Conduct code: define diretrizes de comportamento dos usuários em relação às suas atividades colaborativas no sistema.

- Arbitration and arbitration enforcement: permite solicitar, ao comitê, análise e decisão relacionada a problemas contínuos de má conduta. Utilizado apenas após os outros meios de resolução de disputa falharem.

○ Formal Mediation: permite requisitar, ao conselho, a mediação formal da Wiki, com objetivo de deliberar definitivamente a respeito da disputa em andamento. 
- Change tracking and versioning/History: permite acessar o histórico de alterações realizadas para uma determinada entidade, havendo a possibilidade de reverter as edições feitas.

- Activity/Provenance: permite registrar as alterações feitas pelos usuários, a fim de gratificar os que mais contribuíram com uma determinada porção dos dados ou mesmo na ontologia.

- Blocking policy: permite que os administradores impeçam um determinado usuário ou endereço IP de realizar alterações nas informações do sistema.

- Page protection policy: permite que os administradores coloquem diferentes níveis de proteção em cada página a fim de impedir vandalismo.

- Style Guidelines: são diretrizes de estilo, que definem regras para nomear itens ou outras entidades do sistema.

- Moderator approval: condiciona a contribuição dos usuários à aprovação de um usuário com permissões administrativas.

- Permission configuration for collection creation/deletion: permite que a criação ou remoção de coleções seja configurada para determinados tipos de usuário do sistema.

○ Create User/Edit User Data/Delete User Registry: permite que o administrador tenha o controle sobre os dados de todos os usuários, com autorização para criar novos usuário ou editar dados de usuários anteriormente cadastrados. 